\title{
Stabilization of Continuous-time Hybrid Stochastic Differential Equations by Discrete-time Feedback Control ${ }^{\star}$
}

\author{
Xuerong $\mathrm{MaO}^{\text {a }}$ \\ ${ }^{a}$ Department of Mathematics and Statistics, University of Strathclyde, Glasgow G1 1XH, UK
}

\begin{abstract}
In this paper we are concerned with the mean-square exponential stabilization of continuous-time hybrid stochastic differential equations (also known as stochastic differential equations with Markovian switching) by discrete-time feedback controls. Although the stabilization by continuous-time feedback controls for such equations has been discussed by several authors (see e.g. $[9,15,17,25,26])$, there is so far no result on the stabilization by discrete-time feedback controls. Our aim here is to initiate the study in this area by establishing some new results.
\end{abstract}

Key words: Brownian motion, Markov chain, mean-square exponential stability, discrete-time feedback control.

\section{Introduction}

One of the important issues in the study of hybrid stochastic differential equations (SDEs) is the automatic control, with subsequent emphasis being placed on the analysis of stability $[3,9,19,16,17,21-24,27]$. In particular, [13,14] are two of most cited papers (Google citations 412 and 251,respectively) while [18] is the first book in this area (Google citation 424). This paper is concerned with the mean-square exponential stabilization of the hybrid Itô SDEs by discrete-time feedback controls. Throughout this paper the SEDs are in the Itô sense and we will not mention this any more. The stabilization by continuous-time (regular) feedback controls for such equations has been discussed by several authors e.g. $[9,15,17,25,26]$. Here, given an unstable hybrid SDE in the form of (1) with $u=0$, it is required to find a feedback control $u(x(t), r(t), t)$, based on the current state, so that the controlled system

$$
\begin{aligned}
d x(t) & =(f(x(t), r(t), t)+u(x(t), r(t), t)) d t \\
& +g(x(t), r(t), t) d w(t)
\end{aligned}
$$

becomes stable. Here $x(t) \in R^{n}$ is the state, $w(t)=$ $\left(w_{1}(t), \cdots, w_{m}(t)\right)^{T}$ is an $m$-dimensional Brownian motion and $r(t)$ is a Markov chain (please see Section 2 for the formal definitions). Moreover, the control $u$ is $R^{n}$ -

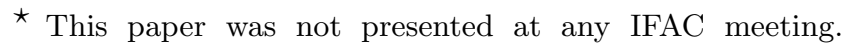
Tel:+441415483669. Fax:+441415483345

Email address: x.mao@strath.ac.uk (Xuerong Mao).
}

valued and, in practice, some of its components are set to be zero if the corresponding components of $d x(t)$ are not affected by $u$. Such a continuous-time feedback control requires continuous observation of the state $x(t)$ for all time $t \geq 0$. However, it is more realistic and costs less in practice if the state is only observed at discrete times, say $0, \tau, 2 \tau, \cdots$, where $\tau>0$ is the duration between two consecutive observations [6]. Accordingly, the feedback control should be designed based on these discrete-time observations, namely the feedback control should be of the form $u(x([t / \tau] \tau), r(t), t)$, where $[t / \tau]$ is the integer part of $t / \tau$. Hence, the stabilization problem becomes to design a discrete-time feedback control $u(x([t / \tau] \tau), r(t), t)$ in the drift part so that the controlled system

$$
\begin{aligned}
d x(t) & =(f(x(t), r(t), t)+u(x([t / \tau] \tau), r(t), t)) d t \\
& +g(x(t), r(t), t) d w(t)
\end{aligned}
$$

becomes stable. To the best knowledge of the author, there is so far no result on this stabilization problem by discrete-time feedback controls, although the corresponding problem for the deterministic differential equations has been studied by many authors (see e.g. $[1,4,5,7,8])$. Our aim here is to initiate the study in this area by establishing some new results. To address the mean-square exponential stability of the controlled system (2), we will relate it with the continuous-time controlled SDE (1). We will show that if the SDE (1) is mean-square exponentially stable, then so is the discrete-time controlled system (2) provided $\tau$ is sufficiently small. But the stabilization problem (1) 
has already been discussed by several authors e.g. $[9,15,17,25,26]$, though the theory could be developed further. In other words, our new theory enables us to transfer our discrete-time controlled problem into the classical (or regular) continuous-time controlled problem. Let us begin to establish our new theory.

\section{Notation and Stabilization Problem}

Throughout this paper, unless otherwise specified, we let $\left(\Omega, \mathcal{F},\left\{\mathcal{F}_{t}\right\}_{t>0}, \mathbb{P}\right)$ be a complete probability space with a filtration $\left\{\mathcal{F}_{t}\right\}_{t \geq 0}$ satisfying the usual conditions (i.e. it is right continuous and $\mathcal{F}_{0}$ contains all $\mathbb{P}$-null sets). Let $w(t)=\left(w_{1}(t), \cdots, w_{m}(t)\right)^{T}$ be an $m$-dimensional Brownian motion defined on the probability space. If $A$ is a vector or matrix, its transpose is denoted by $A^{T}$. If $x \in R^{n}$, then $|x|$ is its Euclidean norm. If $A$ is a matrix, we let $|A|=\sqrt{\operatorname{trace}\left(A^{T} A\right)}$ be its trace norm and $\|A\|=\max \{|A x|:|x|=1\}$ be the operator norm. If $A$ is a systematic matrix $\left(A=A^{T}\right)$, denote by $\lambda_{\min }(A)$ and $\lambda_{\max }(A)$ its smallest and largest eigenvalue, respectively. By $A \leq 0$ and $A<0$, we mean $A$ is non-negative and negative definite, respectively. Denote by $L_{\mathcal{F}_{t}}^{2}\left(R^{n}\right)$ the family of all $\mathcal{F}_{t}$-measurable $R^{n}$-valued random variables $\xi$ such that $E|\xi|^{2}<\infty$. If both $a, b$ are real numbers, then $a \vee b=\min \{a, b\}$ and $a \wedge b=\max \{a, b\}$. Let $r(t), t \geq 0$, be a right-continuous Markov chain on the probability space taking values in a finite state space $S=\{1,2, \cdots, N\}$ with the generator $\Gamma=\left(\gamma_{i j}\right)_{N \times N}$. We assume that the Markov chain $r(\cdot)$ is independent of the Brownian motion $w(\cdot)$. Consider an $n$-dimensional controlled hybrid SDE

$$
\begin{aligned}
d x(t) & =\left(f(x(t), r(t), t)+u\left(x\left(\delta\left(t, t_{0}, \tau\right)\right), r(t), t\right)\right) d t \\
& +g(x(t), r(t), t) d w(t)
\end{aligned}
$$

on $t \geq t_{0}$, with initial data $x\left(t_{0}\right)=x_{0} \in L_{\mathcal{F}_{t_{0}}}^{2}\left(R^{n}\right)$ and $r\left(t_{0}\right)=r_{0} \in M_{\mathcal{F}_{t_{0}}}(S)$ at time $t_{0} \geq 0$. Here $\tau>0$ and

$$
\delta\left(t, t_{0}, \tau\right)=t_{0}+\left[\left(t-t_{0}\right) / \tau\right] \tau
$$

in which $\left[\left(t-t_{0}\right) / \tau\right]$ is the integer part of $\left(t-t_{0}\right) / \tau$. Our aim here is to design the feedback control $u\left(x\left(\delta\left(t, t_{0}, \tau\right)\right), r(t), t\right)$ so that this controlled hybrid SDE becomes mean-square exponentially stable, though the given uncontrolled system (3) with $u=0$ may not be stable. We observe that the feedback control $u\left(x\left(\delta\left(t, t_{0}, \tau\right)\right), r(t), t\right)$ is designed based on the discretetime state observations $x\left(t_{0}\right), x\left(t_{0}+\tau\right), x\left(t_{0}+2 \tau\right), \cdots$, though the given hybrid $\operatorname{SDE}(3)$ with $u=0$ is of continuous-time. In this paper we impose the following standing hypothesis.

Assumption 2.1 Assume that there are positive con- stants $K_{1}, K_{2}, K_{3}$ such that

$$
\begin{aligned}
& |f(x, i, t)-f(y, i, t)| \leq K_{1}|x-y| \\
& |u(x, i, t)-u(y, i, t)| \leq K_{2}|x-y| \\
& |g(x, i, t)-g(y, i, t)| \leq K_{3}|x-y|
\end{aligned}
$$

for all $(x, y, i, t) \in R^{n} \times R^{n} \times S \times R_{+}$. Moreover,

$$
f(0, i, t)=0, \quad u(0, i, t)=0, \quad g(0, i, t)=0
$$

for all $(i, t) \in S \times R_{+}$.

It should be pointed out that condition (6) is for the stability purpose of this paper and condition (5) is for the existence and uniqueness of the solution (see e.g. $[10,11,18,20])$. We also see that these conditions imply the following linear growth condition

$$
|f| \leq K_{1}|x|, \quad|u| \leq K_{2}|x|, \quad|g| \leq K_{3}|x|
$$

for all $(x, i, t) \in R^{n} \times S \times R_{+}$. We also observe that equation (3) is in fact a stochastic differential delay equation (SDDE) with a bounded variable delay. Indeed, if we define the bounded variable delay $\zeta:\left[t_{0}, \infty\right) \rightarrow[0, \tau]$ by

$$
\zeta(t)=t-\left(t_{0}+k \tau\right) \quad \text { for } t_{0}+k \tau \leq t<t_{0}+(k+1) \tau,
$$

for $k=0,1,2, \cdots$, then equation (3) can be written as

$$
\begin{aligned}
d x(t) & =(f(x(t), r(t), t)+u(x(t-\zeta(t)), r(t), t)) d t \\
& +g(x(t), r(t), t) d w(t) .
\end{aligned}
$$

It is therefore known (see e.g. [18]) that under Assumption 2.1, equation (3) has a unique solution $x(t)$ such that $\mathbb{E}|x(t)|^{2}<\infty$ for all $t \geq t_{0}$. To emphasize the role of the initial data, we will denote the solution by $x\left(t ; x_{0}, r_{0}, t_{0}\right)$ and the Markov chain by $r\left(t ; r_{0}, t_{0}\right)$. As equation (3) is a delay SDE, the pair $\left(x\left(t ; x_{0}, r_{0}, t_{0}\right), r\left(t ; r_{0}, t_{0}\right)\right)$ is in general not a Markov process. However, due to the special feature of equation (3), the pair has its Markov property at the discrete times $t_{0}+k \tau(k \geq 0)$. In fact, given $\left(x\left(t_{0}+k \tau ; x_{0}, r_{0}, t_{0}\right), r\left(t_{0}+k \tau ; r_{0}, t_{0}\right)\right)$ at time $t_{0}+k \tau$, the process $\left(x\left(t ; x_{0}, r_{0}, t_{0}\right), r\left(t ; r_{0}, t_{0}\right)\right)$ is uniquely determined for all $t \geq t_{0}+k \tau$ but how it reaches $\left(x\left(t_{0}+\right.\right.$ $\left.\left.k \tau ; x_{0}, r_{0}, t_{0}\right), r\left(t_{0}+k \tau ; r_{0}, t_{0}\right)\right)$ from $\left(x_{0}, r_{0}\right)$ is of no use. The process also has its flow property at the discrete times, namely

$$
\begin{aligned}
& \left(x\left(t ; x_{0}, r_{0}, t_{0}\right), r\left(t ; r_{0}, t_{0}\right)\right) \\
= & \left(x\left(t ; x\left(t_{0}+k \tau\right), r\left(t_{0}+k \tau\right), t_{0}+k \tau\right),\right. \\
& \left.r\left(t ; r\left(t_{0}+k \tau\right), t_{0}+k \tau\right)\right),
\end{aligned}
$$

for all $t \geq t_{0}+k \tau$, where $x\left(t_{0}+k \tau\right)=x\left(t_{0}+k \tau ; x_{0}, r_{0}, t_{0}\right)$ and $r\left(t_{0}+k \tau\right)=r\left(t_{0}+k \tau ; r_{0}, t_{0}\right)$. 
Let us now consider the auxiliary controlled hybrid SDE

$$
\begin{aligned}
d y(t) & =(f(y(t), r(t), t)+u(y(t), r(t), t)) d t \\
& +g(y(t), r(t), t) d w(t)
\end{aligned}
$$

on $t \geq t_{0}$, with initial data $y\left(t_{0}\right)=x_{0} \in L_{\mathcal{F}_{t_{0}}}^{2}\left(R^{n}\right)$ and $r\left(t_{0}\right)=r_{0} \in M_{\mathcal{F}_{t_{0}}}(S)$ at time $t_{0} \geq 0$. The difference between this SDE and the original SDE (3) is that the feedback control here is based on the continuous-time state observation $y(t)$. It is known (see e.g. [18]) that under Assumption 2.1, equation (10) has a unique solution, denoted by $y\left(t ; x_{0}, r_{0}, t_{0}\right)$ such that $\mathbb{E}\left|y\left(t ; x_{0}, r_{0}, t_{0}\right)\right|^{2}<\infty$ for all $t \geq t_{0}$. Assume that we know how to design the control function $u: R^{n} \times S \times R_{+} \rightarrow R^{n}$ for this auxiliary controlled hybrid SDE to be mean-square exponentially stable. (The techniques developed in $[9,15,17,25,26]$, for example, can be used to design the control function $u$ and we will illustrate this in Section 4 below.) We will then show that this same control function also makes the original discrete-time controlled system (3) to be mean-square exponentially stable as long as $\tau$ is sufficiently small (namely we make state observations frequently enough). We therefore assume in this paper that this auxiliary controlled hybrid SDE (10) is mean-square exponentially stable. To be precise, let us state it as an assumption.

Assumption 2.2 Assume that there is a pair of positive constants $M$ and $\gamma$ such that the solution of the auxiliary controlled hybrid SDE (10) satisfies

$$
\mathbb{E}\left|y\left(t ; x_{0}, r_{0}, t_{0}\right)\right|^{2} \leq M \mathbb{E}\left|x_{0}\right|^{2} e^{-\gamma\left(t-t_{0}\right)} \quad \forall t \geq t_{0}
$$

for all $t_{0} \geq 0, x_{0} \in L_{\mathcal{F}_{t_{0}}}^{2}\left(R^{n}\right)$ and $r_{0} \in M_{\mathcal{F}_{t_{0}}}(S)$.

We can now state our main result.

\section{Main Result}

Theorem 3.1 Let Assumptions 2.1 and 2.2 hold. Let $\tau^{*}>0$ be the unique root to the equation

$$
\bar{K}\left(\tau^{*}\right)(4 M)^{\left(2 K_{1}+3 K_{2}+K_{3}^{2}\right) / \gamma}=\frac{1}{2},
$$

where

$\bar{K}(\tau)=\frac{K_{2} M \tau}{\gamma}\left[4 \tau\left(K_{1}^{2}+K_{2}^{2}\right)+2 K_{3}^{2}\right] e^{\left(\gamma+2 K_{1}+3 K_{2}+K_{3}^{2}\right) \tau}$.

If $\tau<\tau^{*}$, then there is a pair of positive constants $\bar{M}$ and $\lambda$ such that the solution of the controlled hybrid SDE (3) satisfies

$$
\mathbb{E}\left|x\left(t ; x_{0}, r_{0}, t_{0}\right)\right|^{2} \leq \bar{M} \mathbb{E}\left|x_{0}\right|^{2} e^{-\lambda\left(t-t_{0}\right)} \quad \forall t \geq t_{0}
$$

for all $t_{0} \geq 0, x_{0} \in L_{\mathcal{F}_{t_{0}}}^{2}\left(R^{n}\right)$ and $r_{0} \in M_{\mathcal{F}_{t_{0}}}(S)$.
To prove this theorem, let us present a lemma but we omit the proof as it is quite standard (see e.g. [12]).

Lemma 3.2 Let Assumptions 2.1 and 2.2 hold. For any initial data $x_{0}, r_{0}, t_{0}$, write $x\left(t ; x_{0}, r_{0}, t_{0}\right)=x(t)$ and $y\left(t ; x_{0}, r_{0}, t_{0}\right)=y(t)$. Then, for all $t \geq t_{0}$,

$$
\mathbb{E}|x(t)-y(t)|^{2} \leq K(\tau) \mathbb{E}\left|x_{0}\right|^{2} e^{\left(2 K_{1}+3 K_{2}+K_{3}^{2}\right)\left(t-t_{0}\right)},
$$

where $K(\tau)=K_{2} M \tau e^{\gamma \tau}\left[4 \tau\left(K_{1}^{2}+K_{2}^{2}\right)+2 K_{3}^{2}\right] / \gamma$.

Proof of Theorem 3.1. Fix initial data $x_{0}, r_{0}, t_{0}$ arbitrarily and write $x\left(t ; x_{0}, r_{0}, t_{0}\right)=x(t)$ and $r\left(t ; r_{0}, t_{0}\right)=r(t)$ simply. For $k=0,1,2, \cdots$, we write $t_{0}+k \tau=t_{k}$, $x\left(t_{0}+k \tau\right)=x_{k}$ and $r\left(t_{0}+k \tau\right)=r_{k}$. Recalling the flow property (9), we see that

$$
x(t)=x\left(t ; x_{k}, r_{k}, t_{k}\right) \quad \forall t \geq t_{k} .
$$

In other words, when $t \geq t_{k}$, we may regard $x(t)$ as the solution of the SDE (3) with initial data $x\left(t_{k}\right)=x_{k}$ and $r\left(t_{k}\right)=r_{k}$ at time $t_{k}$. Let us choose a positive integer $\bar{k}$ such that

$$
\frac{\log (4 M)}{\gamma \tau} \leq \bar{k}<\frac{\log (4 M)}{\gamma \tau}+1
$$

So

$$
2 M e^{-\gamma \bar{k} \tau} \leq \frac{1}{2}
$$

For $i=0,1,2, \cdots$, let $y_{(i+1) \bar{k}}=y\left((i+1) \bar{k} ; x_{i \bar{k}}, r_{i \bar{k}}, t_{i \bar{k}}\right)$. By Assumption 2.2 and (18), we have

$$
\mathbb{E}\left|y_{(i+1) \bar{k}}\right|^{2} \leq M e^{-\gamma \bar{k} \tau} \mathbb{E}\left|x_{i \bar{k}}\right|^{2} \leq \frac{1}{4} \mathbb{E}\left|x_{i \bar{k}}\right|^{2}
$$

On the other hand, by (16), we also have $x_{(i+1) \bar{k}}=x((i+$ 1) $\left.\bar{k} ; x_{i \bar{k}}, r_{i \bar{k}}, t_{i \bar{k}}\right)$. Hence, by Lemma 3.2 ,

$$
\mathbb{E}\left|x_{(i+1) \bar{k}}-y_{(i+1) \bar{k}}\right|^{2} \leq K(\tau) e^{\left(2 K_{1}+3 K_{2}+K_{3}^{2}\right) \bar{k} \tau} \mathbb{E}\left|x_{i \bar{k}}\right|^{2} .
$$

But, by (17),

$$
\begin{aligned}
& e^{\left(2 K_{1}+3 K_{2}+K_{3}^{2}\right) \bar{k} \tau} \leq e^{\left(2 K_{1}+3 K_{2}+K_{3}^{2}\right)(\log (4 M) / \gamma+\tau)} \\
& =e^{\left(2 K_{1}+3 K_{2}+K_{3}^{2}\right) \tau}(4 M)^{\left(2 K_{1}+3 K_{2}+K_{3}^{2}\right) / \gamma}
\end{aligned}
$$

Substituting this into (20) and recalling the definition of $\bar{K}(\tau)$ in the statement of Theorem 3.1, we see that

$$
\begin{aligned}
& \mathbb{E}\left|x_{(i+1) \bar{k}}-y_{(i+1) \bar{k}}\right|^{2} \\
& \leq \frac{1}{2} \bar{K}(\tau)(4 M)^{\left(2 K_{1}+3 K_{2}+K_{3}^{2}\right) / \gamma} \mathbb{E}\left|x_{i \bar{k}}\right|^{2} .
\end{aligned}
$$


By (19) and (21) we therefore have that

$$
\mathbb{E}\left|x_{(i+1) \bar{k}}\right|^{2} \leq\left(\frac{1}{2}+\bar{K}(\tau)(4 M)^{\left(2 K_{1}+3 K_{2}+K_{3}^{2}\right) / \gamma}\right) \mathbb{E}\left|x_{i \bar{k}}\right|^{2} .
$$

Since $\tau<\tau^{*}$ and $\bar{K}(\tau)$ is an increasing function of $\tau$, we see from (12) that

$$
\frac{1}{2}+\bar{K}(\tau)(4 M)^{\left(2 K_{1}+3 K_{2}+K_{3}^{2}\right) / \gamma}<1
$$

Hence may write

$$
\frac{1}{2}+\bar{K}(\tau)(4 M)^{\left(2 K_{1}+3 K_{2}+K_{3}^{2}\right) / \gamma}=e^{-\lambda \bar{k} \tau}
$$

for some $\lambda>0$. It then follows from (22) that

$$
\mathbb{E}\left|x_{(i+1) \bar{k}}\right|^{2} \leq \mathbb{E}\left|x_{i \bar{k}}\right|^{2} e^{-\lambda \bar{k} \tau}
$$

This implies immediately that

$$
\mathbb{E}\left|x_{i \bar{k}}\right|^{2} \leq \mathbb{E}\left|x_{0}\right|^{2} e^{-\lambda i \bar{k} \tau}, \quad \forall i=0,1,2, \cdots
$$

Now, for any $t \geq t_{0}$, there is a unique $i \geq 0$ such that $t_{0}+i \bar{k} \tau \leq t<t_{0}+(i+1) \bar{k} \tau$. By (16), we have

$$
x(t)=x\left(t ; x_{i \bar{k}}, r_{i \bar{k}}, t_{i \bar{k}}\right) .
$$

By the Itô formula and Assumption 2.1, it is easy to show that

$$
\begin{aligned}
& \sup _{t_{i \bar{k}} \leq u \leq t} \mathbb{E}|x(u)|^{2} \leq \mathbb{E}\left|x_{i \bar{k}}\right|^{2} \\
& +\left(2 K_{1}+2 K_{2}+K_{3}^{2}\right) \mathbb{E} \int_{t_{i \bar{k}}}^{t}\left[\sup _{t_{i \bar{k}} \leq u \leq s} \mathbb{E}|x(u)|^{2}\right] d s .
\end{aligned}
$$

for $t \geq t_{i \bar{k}}$. The Gronwall inequality yields

$$
\mathbb{E}|x(t)|^{2} \leq \mathbb{E}\left|x_{i \bar{k}}\right|^{2} e^{\left(2 K_{1}+2 K_{2}+K_{3}^{2}\right)\left(t-t_{i \bar{k}}\right)} \quad \forall t \geq t_{i \bar{k}}
$$

This, together with (24), implies

$$
\begin{aligned}
\mathbb{E}|x(t)|^{2} & \leq \mathbb{E}\left|x_{i \bar{k}}\right|^{2} e^{\left(2 K_{1}+2 K_{2}+K_{3}^{2}\right) \bar{k} \tau} \\
& \leq \mathbb{E}\left|x_{0}\right|^{2} e^{-\lambda i \bar{k} \tau+\left(2 K_{1}+2 K_{2}+K_{3}^{2}\right) \bar{k} \tau} \\
& \leq \bar{M} \mathbb{E}\left|x_{0}\right|^{2} e^{-\lambda\left(t-t_{0}\right)}
\end{aligned}
$$

where $\bar{M}=e^{\lambda \tau+\left(2 K_{1}+2 K_{2}+K_{3}^{2}\right) \bar{k} \tau}$. But this is the required assertion (14). $\square$.

\section{Design of Discrete-time Feedback Control}

The new theory established above enables us to design the discrete-time feedback control for the stabilization problem (3) in two steps: (i) Design the control function $u: R^{n} \times S \times R_{+} \rightarrow R^{n}$ for the auxiliary hybrid SDE (10) to be mean-square exponentially stable.

(ii) Find the unique root $\tau^{*}>0$ to equation (12) and make sure $\tau<\tau^{*}$.

Then the discrete-time control $u\left(x\left(\delta\left(t, t_{0}, \tau\right)\right), r(t), t\right)$ will stabilize the controlled hybrid SDE in the sense of the mean-square exponential stability. Step (i) has its own right of course. In fact, there is an intensive literature in the study of the stabilization problem (10). For example, the stabilization by a continuous-time (regular) feedback control or sliding mode control has been discussed by several authors $[9,15,17,25,26]$. We have no space in this paper to develop in this direction but we will only make use of the existing results to illustrate the two steps above. Due to the page limit, we will only apply the results in [15] to the linear hybrid SDEs for illustrations but leave the other results and nonlinear SDEs to the reader.

Suppose that we are given an $n$-dimensional unstable linear hybrid SDE

$$
d x(t)=A(r(t)) x(t) d t+\sum_{k=1}^{m} B_{k}(r(t)) x(t) d w_{k}(t)
$$

on $t \geq t_{0}$. Here $A, B_{k}$ are mappings from $S \rightarrow R^{n \times n}$ and we will also write $A(i)=A_{i}$ and $B_{k}(i)=B_{k i}$. What we are required is to design a feedback control function, in the form of $u(x, i)=F(i) G(i) x$, in the drift part so that the controlled SDE

$$
\begin{aligned}
d x(t) & =\left[A(r(t)) x(t)+F(r(t)) G(r(t)) x\left(\delta\left(t, t_{0}, \tau\right)\right)\right] d t \\
& +\sum_{k=1}^{m} B_{k}(r(t)) x(t) d w_{k}(t)
\end{aligned}
$$

will be mean-square exponentially stable, where $F$ and $G$ are mappings from $S$ to $R^{n \times l}$ and $R^{l \times n}$, respectively, and we will also write $F(i)=F_{i}$ and $G(i)=G_{i}$. In practice, only one of them is given while the other needs to be designed. They are known as [15]: (i) state feedback: design $F(\cdot)$ when $G(\cdot)$ is given; (ii) output injection: design $G(\cdot)$ when $F(\cdot)$ is given. We will only discuss the case of state feedback. By Theorem 3.1, our first step is to design $F(\cdot)$ for the following controlled hybrid SDE

$$
\begin{aligned}
d y(t) & =[A(r(t))+F(r(t)) G(r(t))] y(t) d t \\
& +\sum_{k=1}^{m} B_{k}(r(t)) y(t) d w_{k}(t)
\end{aligned}
$$

to be mean-square exponentially stable. Assume that for some number $\gamma>0$, we can find a set of solutions $Q_{i} \in R^{n \times n}$ and $Y_{i} \in R^{n \times l}(i \in S)$, with $Q_{i}=Q_{i}^{T}>0$, 
to the following linear matrix inequalities (LMIs)

$$
\begin{gathered}
Q_{i} A_{i}+Y_{i} G_{i}+A_{i}^{T} Q_{i}+G_{i}^{T} Y_{i}^{T}+\sum_{k=1}^{m} B_{k i}^{T} Q_{i} B_{k i} \\
+\sum_{j=1}^{N} \gamma_{i j} Q_{j}+\gamma Q_{i} \leq 0
\end{gathered}
$$

Set $F_{i}=Q_{i}^{-1} Y_{i}$. Then, in the same way as [15, Theorem 3.2] was proved, we can show that the solution of equation (28) satisfies

$$
\mathbb{E}\left|y\left(t ; x_{0}, r_{0}, t\right)\right|^{2} \leq M \mathbb{E}\left|x_{0}\right|^{2} e^{-\gamma\left(t-t_{0}\right)}, \quad \forall t \geq t_{0}
$$

for all $t_{0} \geq 0, x_{0} \in L_{\mathcal{F}_{t_{0}}}^{2}\left(R^{n}\right)$ and $r_{0} \in M_{\mathcal{F}_{t_{0}}}(S)$, where

$$
M=\frac{\max _{i \in S} \lambda_{\max }\left(Q_{i}\right)}{\min _{i \in S} \lambda_{\min }\left(Q_{i}\right)} .
$$

That is, Assumption 2.2 is fulfilled. It is obvious that Assumption 2.1 is also fulfilled with

$$
\begin{array}{r}
K_{1}=\max _{i \in S}\left\|A_{i}\right\|, K_{2}=\max _{i \in S}\left\|Q_{i}^{-1} Y_{i} G_{i}\right\|, \\
K_{3}=\max _{i \in S} \sqrt{\sum_{k=1}^{m}\left\|B_{k i}\right\|^{2}} .
\end{array}
$$

By Theorem 3.1, we therefore obtain the following useful corollary.

Corollary 4.1 Assume that for some number $\gamma>0$, there is a set of solutions $Q_{i} \in R^{n \times n}$ and $Y_{i} \in R^{n \times l}$ $(i \in S)$, with $Q_{i}=Q_{i}^{T}>0$, to the LMIs (29). Let $M, K_{1}, K_{2}, K_{3}$ be defined by (31) and (32). Let $\tau^{*}>0$ be the unique root to equation (12). If we set $F_{i}=Q_{i}^{-1} Y_{i}$ $(i \in S)$ and make sure that $\tau<\tau^{*}$, then the controlled hybrid SDE (27) is mean-square exponentially stable.

Example 4.2 Consider the linear hybrid SDE

$$
d x(t)=A(r(t)) x(t) d t+B(r(t)) x(t) d w(t)
$$

on $t \geq t_{0}$. Here $w(t)$ is a scalar Brownian motion; $r(t)$ is a Markov chain on the state space $S=\{1,2\}$ with the generator

$$
\Gamma=\left[\begin{array}{rr}
-1 & 1 \\
1 & -1
\end{array}\right]
$$

and the system matrices are

$$
\begin{aligned}
& A_{1}=\left[\begin{array}{rr}
1 & -1 \\
1 & -5
\end{array}\right], \quad A_{2}=\left[\begin{array}{rr}
-5 & -1 \\
1 & 1
\end{array}\right], \\
& B_{1}=\left[\begin{array}{rr}
1 & 1 \\
1 & -1
\end{array}\right], \quad B_{2}=\left[\begin{array}{rr}
-1 & -1 \\
-1 & 1
\end{array}\right] .
\end{aligned}
$$

This hybrid SDE is not mean square exponentially stable. Let us now design a discrete-time state feedback control to stabilize the system. Assume that the controlled hybrid SDE has the form

$$
\begin{aligned}
d x(t) & =\left[A(r(t)) x(t)+F(r(t)) G(r(t)) x\left(\delta\left(t, t_{0}, \tau\right)\right)\right] d t \\
& +B(r(t)) x(t) d w(t),
\end{aligned}
$$

where

$$
G_{1}=(1,0), \quad G_{2}=(0,1) .
$$

Applying Corollary 4.1, we can show that if we set

$$
F_{1}=\left[\begin{array}{c}
-10 \\
0
\end{array}\right], \quad F_{2}=\left[\begin{array}{c}
0 \\
-10
\end{array}\right],
$$

and make sure that $\tau<\tau^{*}=0.0000308$, then the discrete-time controlled hybrid SDE (34) is mean-square exponentially stable. The computer simulation (Figure 4.1) supports this result clearly.
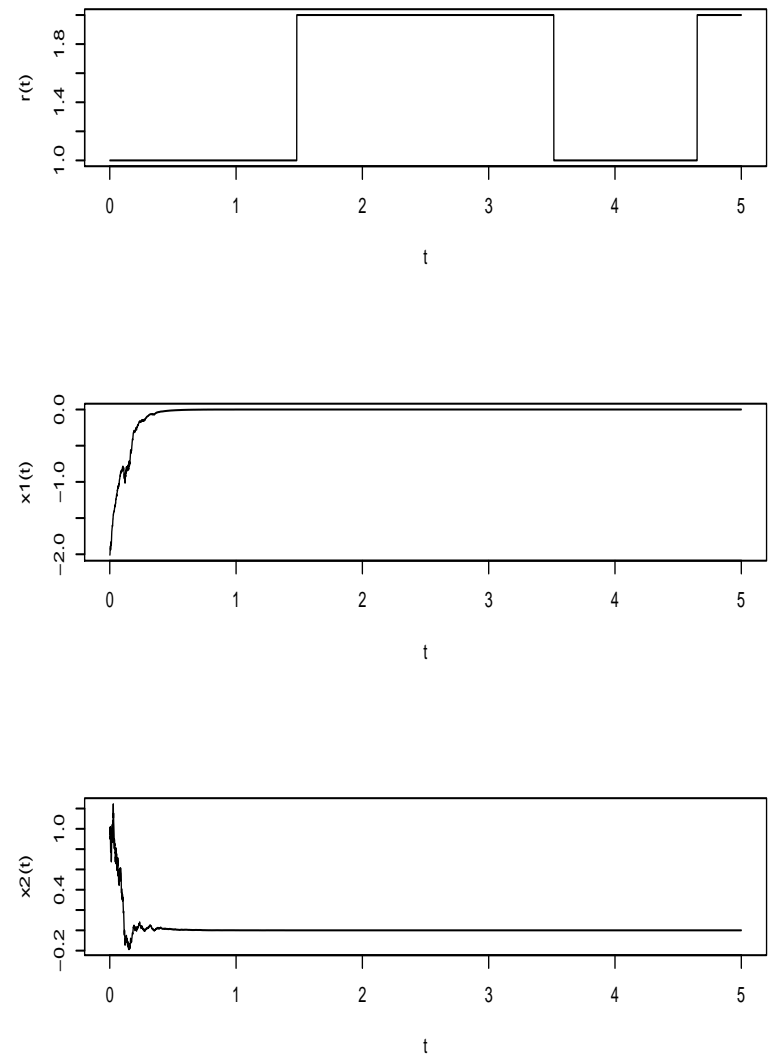

Figure 4.1: Computer simulation of the paths of $r(t), x_{1}(t)$ and $x_{2}(t)$ for the discrete-time controlled hybrid SDE (34) with $\tau=10^{-5}$ using the Euler-Maruyama method with step size $10^{-6}$ and initial values $r(0)=1, x_{1}(0)=-2$ and $x_{2}(0)=1$. 


\section{Conclusions and Further Comments}

In this paper we have shown clearly that unstable hybrid SDEs can be stabilized by the discrete-time feedback controls.

We should also point out that to make our theory more understandable as well as to avoid complicated notations, we have only considered the controlled hybrid SDE (3), where the discrete-time control is designed in the drift part. It is useful and interesting to consider the case where the discrete-time control is designed in the diffusion part, or different discrete-time controls are designed in the drift and diffusion parts. However, due to the page limit here, we will report these results elsewhere.

\section{Acknowledgements}

The author would like to thank the editors and referees for their helpful suggestions and comments. He would also like to thank the EPSRC, the Royal Society of Edinburgh, the London Mathematical Society, the Edinburgh Mathematical Society, the National Natural Science Foundation of China (grants 71073023) for their financial support.

\section{References}

[1] Allwright, J.C., Astolfi, A. and Wong, H.P., A note on asymptotic stabilization of linear systems by periodic, piecewise constant, output feedback, Automatica 41(2) (2005), 339-344.

[2] Anderson, W.J., Continuous-Time Markov Chains, Springer, New York, 1991.

[3] Basak, G.K., Bisi, A. and Ghosh, M.K., Stability of a random diffusion with linear drift, J. Math. Anal. Appl. 202 (1996), 604-622.

[4] Ebihara, Y., Yamaguchi, J. and Hagiwara, T., Periodically time-varying controller synthesis for multiobjective $\mathrm{H}_{2} / \mathrm{H}_{\infty}$ control of discrete-time systems and analysis of achievable performance, Systems Control Lett. 60(9) (2011), 709-717.

[5] Chammas, A.B. and Leondes, C.T., On the finite time control of linear systems by piecewise constant output feedback, Internat. J. Control 30(2) (1979), 227-234.

[6] Chen,T.W. and Francis, B., Optimal SampledData Control Systems, Springer-Verlag, London, 1995.

[7] Hagiwara,T. and Araki, M., Design of stable state feedback controller based on the multirate sampling of the plant output, IEEE Trans. Automat. Control 33(9) (1988), 812819.

[8] Hagiwara,T. and Araki, M., On preservation of strong stabilizability under sampling. IEEE Trans. Automat. Control 33(11) (1988), 1080-1082.

[9] Ji, Y. and Chizeck, H.J., Controllability, stabilizability and continuous-time Markovian jump linear quadratic control, IEEE Trans. Automat. Control 35 (1990), 777-788.

[10] Mao, X., Stability of Stochastic Differential Equations with Respect to Semimartingales, Longman Scientific and Technical, 1991.
[11] Mao, X., Exponential Stability of Stochastic Differential Equations, Marcel Dekker, 1994.

[12] Mao X., Stochastic Differential Equations and Their Applications, 2nd Edition, Chichester: Horwood Pub., 2007.

[13] Mao, X., Stability of stochastic differential equations with Markovian switching, Sto. Proc. Their Appl. 79 (1999), 4567.

[14] Mao, X., Exponential stability of stochastic delay interval systems with Markovian switching, IEEE Trans. Auto. Control 47(10) (2002), 1604-1612.

[15] Mao, X., Lam, J. and Huang, L., Stabilisation of hybrid stochastic differential equations by delay feedback control, Systems \& Control Letters 57 (2008), 927-935.

[16] Mao, X., Matasov, A. and Piunovskiy, A.B., Stochastic differential delay equations with Markovian switching, Bernoulli 6(1) (2000), 73-90.

[17] Mao, X., Yin, G. and Yuan, C., Stabilization and destabilization of hybrid systems of stochastic differential equations, Automatica 43 (2007), 264-273.

[18] Mao, X. and Yuan, C., Stochastic Differential Equations with Markovian Switching, Imperial College Press, 2006.

[19] Mariton, M., Jump Linear Systems in Automatic Control, Marcel Dekker, 1990.

[20] Mohammed, S.-E.A., Stochastic Functional Differential Equations, Longman Scientific and Technical, 1986.

[21] Shaikhet, L., Stability of stochastic hereditary systems with Markov switching, Theory of Stochastic Processes 2(18) (1996), 180-184

[22] Shi, P., Mahmoud, M.S., Yi, J. and Ismail, A., Worst case control of uncertain jumping systems with multi-state and input delay information, Information Sciences 176 (2006), 186-200.

[23] Sun, M., Lam, J., Xu, S. and Zou, Y., Robust exponential stabilization for Markovian jump systems with modedependent input delay, Automatica 43 (2007), 1799-1807.

[24] Wei, G., Wang, Z., Shu, H. and Fang, J., Robust $H_{\infty}$ control of stochastic time-delay jumping systems with nonlinear disturbances, Optim. Control Appl. Meth. 27 (2006), 255271 .

[25] Wu, L., Shi, P. and Gao, H., State estimation and sliding mode control of Markovian jump singular systems, IEEE Trans on Automatic Control 55(5) (2010), 1213-1219.

[26] Wu, L., Su, X. and Shi, P., Sliding mode control with bounded $L_{2}$ gain performance of Markovian jump singular time-delay systems, Automatica 48(8) (2012), 1929-1933.

[27] Yue, D. and Han, Q., Delay-dependent exponential stability of stochastic systems with time-varying delay, nonlinearity, and Markovian switching, IEEE Trans. Automat. Control 50 (2005), 217-222. 